\section{FRI0525 ASSOCIATION OF DYSMOBILITY SYNDROME WITH FRACTURE RISK IN THE MROS COHORT}

B. Buehring ${ }^{1,2}$, B.L. Lewis ${ }^{1}$, K.E. Hansen ${ }^{1}$, S.R. Cummings ${ }^{3}$, N.E. Lane ${ }^{4}$ N. Binkley ${ }^{1}$, K.E. Ensrud ${ }^{5}$, P.M. Cawthon ${ }^{3} .{ }^{1}$ Department of Medicine, University of Wisconsin-Madison, Madison, United States; ${ }^{2}$ Rheumazentrum Ruhrgebiet, Herne, Germany; ${ }^{3}$ California Pacific Medical Center Research Institute, San Francisco; ${ }^{4}$ University of California - Davis, Sacramento; ${ }^{5}$ University of Minnesota, Minneapolis, United States

Background: Osteoporosis, obesity and sarcopenia are risk factors for fractures and their combination has a negative effect on musculoskeletal health (MSKH). We proposed a score-based approach to define this combination as "dysmobility syndrome (DS)". DS increases mortality in the NHANES cohort but no data exist on fracture risk. The most widely used fracture risk calculator, the WHO FRAX ${ }^{\circledR}$ tool, does not include several measures of MSKH such as physical function, muscle mass or falls. In this analysis of the Osteoporotic Fractures in Men (MrOS) cohort, we examine whether individuals with impaired MSKH/DS have a higher incidence of fragility fractures and whether this composite score confers additional risk for fracture, beyond risk estimates provided by FRAX ${ }^{\circledR}$.

Objectives: In this analysis of the Osteoporotic Fractures in Men (MrOS) cohort, we examine whether individuals with impaired MSKH/DS have a higher incidence of fragility fractures and whether this composite score confers additional risk for fracture, beyond risk estimates provided by FRAX ${ }^{\circledR}$

Methods: The MrOS cohort was utilized in this study. The score-based approach to define DS includes six factors with one point assigned to each: appendicular lean mass/height2 $<7.26 \mathrm{~kg} / \mathrm{m}^{2}$, body fat $>30 \%$, T-score $\leq-2.5$, grip strength $<30$ $\mathrm{kg}$, gait speed $<1.0 \mathrm{~m} / \mathrm{s}$, and falls in last 12 months. A score $\geq 3$ indicated DS. We use odds ratios and cox proportional hazard models to analyze risks of major osteoporotic fracture (MOF). Men were censored at the time of fracture or last follow up. We determined the hazards of fracture using presence of dysmobility syndrome, the FRAX ${ }^{\circledR}$ score, and the FRAX ${ }^{\circledR}$ score in quartiles. We used the program R (www.r project.org) to perform all analyses.

Results: 5827 men ages $74 \pm 6$ years with a mean BMl of $27.4 \pm 3.8 \mathrm{~kg} / \mathrm{m}^{2}$ had complete data necessary for this analysis. 391 males $(6.7 \%)$ met criteria for DS. $571(10 \%)$ experienced a MOF including $245(4 \%)$ hip fractures. DS increased the hazards of major osteoporotic ( $\mathrm{HR} 3.31,95 \% \mathrm{Cl}, 2.58,4.23$ ) and hip (HR 3.48 , $95 \% \mathrm{Cl} 2.41,5.03)$ fractures. In adjusted models, DS and elevated FRAX ${ }^{\circledR}$ risk each increased the hazards of major osteoporotic and hip fracture. Interaction models showed no significant interaction between the presence of DS and FRAX ${ }^{\circledR}$ score for major osteoporotic $(p=0.184)$ or hip $(p=0.177)$ fractures.

Table 1. Cox Proportional Hazard Models for Fracture

\begin{tabular}{lcc}
\hline Models & $\begin{array}{c}\text { Major Osteoporotic Fracture } \\
571 \text { events } \\
\text { Hazard Ratio }(95 \% \mathrm{Cl})\end{array}$ & $\begin{array}{c}\text { Hip Fracture } \\
245 \text { events } \\
\text { Hazard Ratio }(95 \% \mathrm{Cl})\end{array}$ \\
\hline Dysmobility Index & $3.31(2.59,4.23)$ & $3.48(2.41,5.03)$ \\
FRAX Score above treatment threshold & $3.56(2.51,5.07)$ & $5.52(4.28,7.11)$ \\
Dysmobility Index & $3.14(2.45,4.02)$ & $2.21(1.52,3.21)$ \\
and FRAX Score & $3.17(2.22,4.51)$ & $5.14(3.97,6.65)$ \\
\hline
\end{tabular}

$\mathrm{Cl}$, Confidence Interval.

Conclusions: DS was associated with increased MOF fracture incidence even after adjusting for quartiles of $F R A X^{\circledR}$ risk in this cohort of older men. Our study suggests that using a composite assessment of $\mathrm{MSKH}$ in addition to already available tools such as FRAX ${ }^{\circledR}$ may improve identification of individuals at high fracture risk. Additional analyses are necessary to examine whether this approach can better distinguish between those who will fracture and who will not and whether the results can be reproduced in women.

Disclosure of Interest: None declared

DOI: 10.1136/annrheumdis-2017-eular.2790

\section{FRI0526 ANTI-OSTEOPOROTIC THERAPY DECREASE CANCER RISK IN PATIENTS WITH OSTEOPOROTIC VERTEBRAL FRACTURES}

Y.-C. Chen, C.-H. Ko. Rheumatology, Kaohsiung Chang Gung Memorial Hospital, Kaohsiung, Taiwan, Province of China

Background: Some studies had looked at the long-term effect of anti-osteoporotic therapy on risks of different cancers in the general population. But epidemiological studies have consistently reported a reduced risk of breast cancer in bisphosphonate users. so there is little data to establish definitive conclusions. Objectives: This study aimed to determine if anti-osteoporotic therapy can influence cancer risk in patients with osteoporotic vertebral fracture.

Methods: This retrospective study reviewed of cases of osteoporosis patients with acute vertebral fractures between 2001 and 2015. Anti-osteoporotic therapy were recorded (alendronate, ibandronate, zolendronic acid, raloxifen, teriparatide, denosumab). We followed these patients to develop cancer. All associated co-morbidities were recorded. Cox regression analysis were performed.

Results: There were 1128 patients with acute vertebral fractures were enrolled, among them 693 patients accepted anti-osteoporotic therapy, 432 were not. The mean age of anti-osteoporotic therapy was $73.86 \pm 7.52$, while the age of nonanti-osteoporotic therapy was $72.82 \pm 10.92(p=0.059) .15(2.2 \%)$ patients of anti-osteoporotic therapy developed cancer, while $24(5.6 \%)$ patients of non- antiosteoporotic therapy developed cancer $(p=0.004)$. After adjusting for potential confounders, patients with antiosteoporotic therapy still had a lower cancer risk $(p=0.038$; HR: $0.428,95 \% \mathrm{Cl}: 0.192 \sim 0.955)$. The cancer risk also increased among smokers $(p=0.002$; HR: $10.505 ; 95 \% \mathrm{Cl}: 2.375 \sim 46.462)$.

Table 1. Multivariable cox regression analysis of the hazard ratios for cancer

\begin{tabular}{lcccc}
\hline & Regression coefficient & SE & P value & HR (95.CI) \\
Anti-osteoporosis & -0.848 & 0.409 & 0.038 & $0.428(0.192-0.955)$ \\
Gender & -0.693 & 0.584 & 0.236 & $0.500(0.159-1.572)$ \\
Age & -0.029 & 0.016 & 0.071 & $0.972(0.942-1.002)$ \\
Smmoking & 2.352 & 0.759 & 0.002 & $10.505(2.375-46.462)$ \\
liver disease & 2.012 & 0.487 & 0.001 & $7.477(2.880-19.412)$ \\
Kidney disease & -0.338 & 1.224 & 0.783 & $0.713(0.065-7.859)$ \\
Cardiovascular disease & 0.856 & 0.677 & 0.206 & $2.355(0.624-8.882)$ \\
Pulmonary disease & 0.771 & 0.597 & 0.197 & $2.162(0.671-6.971)$ \\
\hline
\end{tabular}

Abbreviations: HR, hazard ratio; SE, standard error.

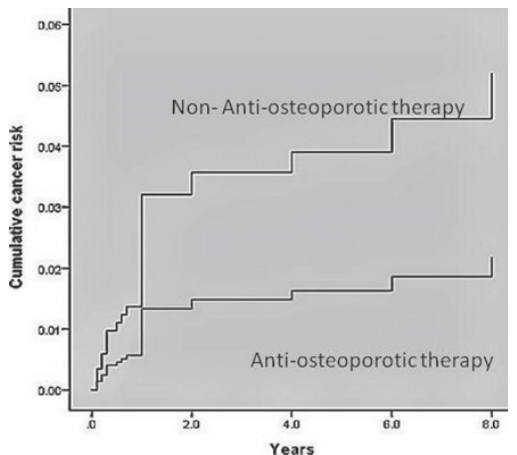

Conclusions: In this study, anti-osteoporotic therapy decrease cancer risk. So we could safely use these drugs in osteoporotic management.

References:

[1] British Journal of Cancer (2013) 109, 795-806.

Acknowledgements: We thanked Kaohsiung Chang Gang Memorial hospital for data support.

Disclosure of Interest: None declared

DOI: 10.1136/annrheumdis-2017-eular.3820

\section{FRI0527 LACUNARITY OF TRABECULAR BONE MICROARCHITECTURE, TBL $\beta$, AS A PREDICTOR OF BONE FRAGILITY FRACTURE AND POTENTIAL INDEX OF OSTEOPOROSIS TREATMENT EFFICACY. THE LOTO STUDY}

A. Zaia ${ }^{1}$, R. Rossi ${ }^{2}$, R. Galeazzi ${ }^{2}$, P. Scendoni ${ }^{3} .{ }^{1}$ Scientific Direction; ${ }^{2}$ Medical Imaging and Services, INRCA Italian National Research Center on Aging, Ancona; ${ }^{3}$ Rheumatology Division, INRCA Italian National Research Center on Aging, Fermo, Italy

Background: Bone mineral density (BMD) by dual-energy X-ray absortiometry (DXA) is still the routinely diagnostic approach for osteoporosis. However, BMD alone is not a good predictor of fracture risk while bone microarchitecture emerges as a determinant of bone fragility independent of BMD. High-resolution magnetic resonance imaging $(\mathrm{MRI})$ represents an effective tool for in vivo characterization of trabecular bone microarchitecture (TBA) by noninvasive/nonionizing methods. Nevertheless, texture analysis is not used in clinical practice because of the large number of parameters to be calculated and analyzed.

We previously developed a MRI method to provide one parameter (TBL $\beta$ ) sensitive to TBA changes in aging and osteoporosis ${ }^{1}$. Fractal lacunarity was chosen for TBA texture analysis as it is able to describe bone network discontinuity and sizes of bone marrow spaces, whose changes are index of increased fracture risk. Objectives: Clinical validation of the method as a new tool useful in early diagnosis of osteoporotic fracture risk is the strategic aim of LOTO study. One major objective is to verify the potential of TBL $\beta$ in discriminating patients with $(\mathrm{VF}+)$ and without (VF-) vertebral fractures. TBL $\beta$ as a potential marker of osteoporosis treatment efficacy is also investigated.

Methods: An observational, cross-sectional and prospective study on over50s women at risk for bone fragility fractures was designed to validate the method ${ }^{2}$ Sample size was estimated equal to 280 osteopenic/osteoporotic women with/without prior vertebral bone fragility fractures. The main outcome measure is $T B L \beta$ as an index of osteoporotic fracture risk. It is calculated by a software prototype of the gray-scale version of our method on $L 4$ axial images acquired by 1.5T MRI spin-echo multislice technique ${ }^{3}$.

Results: A complete set of baseline recording, including DXA-BMD, conventional column Rx morphometry, and lumbar spine MRI-spin echo images for TBA characterization, was obtained for 279 out of 309 subjects eligible for the study. Prevalent VF were found in $31.5 \%$ subjects, $47.7 \%$ of which defined osteopenic at lumbar spine by DXA-BMD and $67 \%$ younger than 65 years. Baseline results from ROC analysis show that the contribution of TBA degeneration (TBL $\beta=40$ ) to prevalent fractures is statistically higher $(p=0.032)$ than BMD (T-score $=-2.5)$. TBL $\beta$ results (Table 1) show that the proposed method is able to discriminate between 